\title{
CONSIDERACIONES IMPORTANTES PARA LA GESTIÓN FINANCIERA Y PLANIFICACIÓN ESTRATÉGICA
}

\section{IMPORTANT CONSIDERATIONS FOR FINANCIAL MANAGEMENT AND STRATEGIC PLANNING}

\author{
Maldonado-Escandón Andrea ${ }^{1 *}$; Zambrano-Loor Rita²; Bernal-Álava Ángel ${ }^{3}$ \\ ${ }^{1}$ Universidad Laica Eloy Alfaro de Manabí, ULEAM. Manta, Ecuador. \\ ${ }^{2}$ Docente de la Universidad Laica Eloy Alfaro de Manabí, ULEAM. Manta, Ecuador. \\ ${ }^{3}$ Docente de la Universidad Estatal del Sur de Manabí, UNESUM. Jipijapa, Ecuador
}

*Correo: ancemes-25@gmail.com

\begin{abstract}
Resumen
La gestión financiera podría definirse como una herramienta que ayuda a reducir recursos en una organización a través de la toma de decisiones, por otra parte, la planificación estratégica corporativa permite establecer objetivos asignando un presupuesto y una fecha determinada para cumplirlos, esto conlleva a que los miembros de la organización se comprometan y cumplan cada uno de sus roles. El objetivo del presente manuscrito es revisar y contrastar los aspectos relevantes de la gestión financiera y la planificación estratégica. Cabe señalar que dentro de una organización sino se cuenta con la información pertinente al momento de tomar decisiones, existe el riesgo de que las actividades no salgan como se lo esperaba y de que ocurra algo desfavorable. Todo cambio en una organización genera nuevas oportunidades que le permitan lograr una estabilidad y reconocimiento en su mercado. Se concluye que, al no aplicar herramientas de gestión estratégica, la empresa tiende a no mantener objetivos acordes a su necesidad actual, por ello, es necesario que se aplique una planificación estratégica corporativa, planteando metas y objetivos que aseguren su éxito empresarial.
\end{abstract}

Palabras clave: gestión administrativa, empresa, planificación, finanzas.

\begin{abstract}
Financial management could be defined as a tool that helps reduce resources in an organization through decision-making, on the other hand, corporate strategic planning allows establishing objectives by assigning a budget and a specific date to meet them, this leads to the members of the organization commit and fulfill each of their roles. The objective of this manuscript is to review and contrast the relevant aspects of financial management and strategic planning. It should be noted that within an organization if the relevant information is not available when making decisions, there is a risk that the activities will not go as expected and that something unfavorable will happen. Any change in an organization generates new opportunities that allow it to achieve stability and recognition in its market. It is concluded that, by not applying strategic management tools, the company tends not to maintain objectives according to its current need, therefore, it is necessary to apply corporate strategic planning, setting goals and objectives that ensure its business success.
\end{abstract}

Keywords: administrative management, company, planning, finance.

Información del manuscrito:

Fecha de recepción: 09 de mayo de 2017

Fecha de aceptación: 27 de junio de 2017

Fecha de publicación: 10 de julio de 2017 


\section{Introducción}

Las organizaciones deben presentar retos importantes en la ejecución de sus operaciones, entre ellas administrar y gestionar de mejor forma los recursos monetarios, que la empresa gestione sus recursos y además de ello aplique una planificación estratégica es lo que le definiría como una organización que está siendo eficiente y eficaz en sus procesos. Muchos empresarios 0 emprendedores al iniciar sus negocios piensan tan solo en estrategias para incrementar sus recursos, olvidándose que existen otros elementos importantes como conocer su entorno, evaluar factores internos y externos que les permitan conocer su mercado competitivo (Castaño-Ramírez, 2011).

Actualmente, el entorno en aspectos políticos, sociales, tecnológicos y económicos ha sido un poco turbulento. Estos cambios afectan a las organizaciones, por lo que se ven obligadas a evaluar la respuesta que presentan ante dichos cambios, con el fin de no afectar la rentabilidad de la misma, por ello es fundamental que las entidades realicen una planificación estratégica corporativa que permita establecer objetivos, metas y estrategias para responder a su entorno (Gutiérrez- Zarabanda, 2013).

Aplicar una correcta gestión financiera en una empresa, implica aspectos orientados hacia la consecución y obtención de los recursos eficientemente. Para evaluar el entorno se considera fundamental utilizar herramientas estratégicas que permitan obtener resultados a través del enfoque planificativo, encontrándose estrechamente vinculada con la planificación estratégica y proporcionando a la empresa un marco referencial para el correcto funcionamiento de la gestión financiera, por lo tanto, la no planificación torna a la organización sensible al entorno.

La gestión financiera podría definirse como una herramienta que ayuda a reducir recursos en una organización a través de la toma de decisiones, por otra parte, la planificación estratégica corporativa permite establecer objetivos asignando un presupuesto y una fecha determinada para cumplirlos, esto conlleva a que los miembros de la organización se comprometan y cumplan cada uno de sus roles 
(Luna-Carreño, 2016). El objetivo del presente manuscrito es revisar y contrastar los aspectos relevantes de la gestión financiera.

\section{Gestión financiera}

Haro de Rosario y Rosario, (2017) con respecto a la definición afirma lo siguiente: una de las subfunciones más destacables es la gestión del circulante, que se centra en la gestión, la situación financiera y la planificación financiera de las decisiones de inversión y financiación que corresponden a cuestiones de funcionamiento o a corto plazo. La gestión de circulante recibe la denominación de finanzas operativas y la de gestión financiera (pp.7).

La gestión financiera ayuda a una organización a tomar decisiones para asignar de mejor manera los recursos disponibles, de esta forma podrán determinar sus necesidades, y el empresario contará con información suficiente que permitirá analizar y considerar la mejor opción para invertir o realizar cambios en su empresa.

Desde un punto de vista financiero el objetivo general de la empresa es maximizar la rentabilidad total de los fondos propios, esto es, maximizar la riqueza de los propietarios de la empresa. Ahora bien, dentro de la dirección financiera o gestión financiera, tal y como se ha dicho, se distinguen dos niveles de actuación. La gestión financiera a largo plazo (finanzas estructurales), que se ocupa de las decisiones de inversión y financiación a largo plazo y está orientada a alcanzar objetivos de rentabilidad y riesgo. Mientras que la gestión financiera a corto plazo (finanzas operativas) comprende el análisis de las decisiones de inversión y financiación a corto plazo y está presidida, fundamentalmente, por objetivos de rentabilidad $y$ liquidez (Haro de Rosario \& Rosario, 2017, p.10).

Es por ello que se puede mencionar que la gestión financiera dentro de una organización funciona como una herramienta que provee varios puntos de vista, que permite el análisis, diagnóstico e incide en la toma de decisiones, definiendo metas y objetivos que se desean obtener y preparando a la organización para adecuarse a los cambios del entorno competitivo. 


\subsection{Importancia}

La gestión financiera es muy importante dentro de una organización porque le permite resolver los temas relacionados a rentabilidad, de esta forma podrá generar recursos oportunos que le permitan ser más eficientes abarcando territorio en el mercado local. Por tanto, la gestión financiera operativa (o de capital de trabajo) se convierte en un instrumento para la obtención de mejores resultados en el orden económico y financiero. Desde el punto de vista financiero, la adecuada gestión de capital de trabajo se traduce, en su aporte al desarrollo empresarial, en liquidez y rentabilidad. Las utilidades netas obtenidas luego del pago de impuestos tendrán dos fines específicos: su retención para llevar a cabo el proceso de crecimiento y desarrollo sostenido de la organización; o su repartición como dividendos a los dueños de la organización (Álvarez-Hernández de Alba et al., 2014, pp. 161). Para garantizar el cumplimiento de los objetivos y alcanzar los ideales del empresario, es necesario, aplicar herramientas de gestión que aseguren el desarrollo de la empresa, entre las cuales se pueden mencionar el F.O.D.A Estratégico, las cinco fuerzas de PORTER o el análisis PEST.

\subsection{El papel de la gestión financiera}

En una organización es necesario que se enfoquen claramente los procedimientos o mecanismos que se utilizan para obtener fondos y de esta forma generar un desarrollo empresarial, por ello se considera necesario que dentro de las estrategias organizacionales se mantenga la venta de acciones a inversores, bonos, e inversiones en otras empresas ya sean estas relacionadas o no. Por ello es común que en las organizaciones existan desequilibrios en aspectos financieros, puesto que al no contar con una respectiva planificación no se conoce con certeza como responder antes los cambios en su entorno.

\section{Consideraciones sobre} liquidez, rentabilidad y riesgo Dentro de todos los aspectos financieros, en la gestión financiera existen tres elementos que merecen una especial consideración, como son liquidez, rentabilidad y riesgo. 


\subsection{Rentabilidad}

La rentabilidad es un concepto relacionado a crecimiento, cuando una empresa es rentable es porque está generando ingresos monetarios, pero hay muchos factores a considerar dentro de este concepto.

"El éxito de cualquier empresa se mide mediante la rentabilidad de su actividad" (Carrasco-Zújar \& Pallerola-Comamala, 2014), por lo que existe una relación estrecha entre los ingresos y los costos en una organización, para así determinar qué tan rentable está siendo el negocio.

\subsection{Liquidez}

"Se considera que una empresa con liquidez es solvente pero no siempre una empresa solvente posee liquidez" (Nava-Rosillón, 2014). Cuando se habla de liquidez es cuando una organización mantiene el efectivo para cubrir todas sus obligaciones, por ello la autora citada hace referencia a que tener liquidez significa cumplir con los compromisos y tener solvencia refleja la disponibilidad que posee para pagar esos compromisos.

\subsection{Riesgo}

Dentro de una organización sino se cuenta con la información pertinente al momento de tomar decisiones, existe el riesgo de que las actividades no salgan como se lo esperaba y de que ocurra algo desfavorable.

"Los inversionistas tienen como principal meta obtener beneficios por la inversión efectuada a la empresa. El rendimiento de éstas debe ser el suficiente para gratificar a los inversionistas una tasa de rendimiento esperada de acuerdo al riesgo de inversión" (Morales \& Morales, 2014).

\section{Conceptualización de la planificación estratégica}

La planificación estratégica es un proceso socio-político y científico orientado por objetivos de transformación cuanti-cualitativos de un sector particular de la realidad. Este proceso articula dos dimensiones de modo dialéctico: la reflexión y la acción, cuyo nexo lo constituye la evaluación, como proceso permanente de conocimiento, revisión, aprendizaje y retroalimentación. Entre la reflexión y la acción media la toma de 
decisiones, es decir definir sobre el qué y de qué manera se llevará algo a la práctica (Rodríguez, 2016).

La planificación estratégica permite visualizar las oportunidades $y$ amenazas que tiene la empresa, que a su vez permiten orientar $y$ direccionar a todo el equipo de trabajo, logrando con ello el desarrollo empresarial a través de estrategias enfocadas en cumplir la misión de la entidad.

\subsection{Importancia}

Según lo mencionado por MoralesCastro \& Morales-Castro (2014) la importancia de la planeación estratégica radica en establecer los objetivos que la empresa desea conseguir a largo plazo, y se convierten en la guía para desarrollar los planes de acción de mediano y largo plazo. En sí, la planificación permite que la empresa se trace un camino preciso para alcanzar los objetivos, conociendo como llegar y que procedimientos realizar para cumplirlos, sirviendo esto como herramienta para el plan de acción que se encuentra estructurado por metas con su respectiva fecha de cumplimiento.

\subsection{Objetivos de la planificación estratégica}

Como lo indica Flores (2013) el objetivo de la planificación estratégica no es solo planear sino de formular e implementar nuevas actividades que a su vez implican recursos humanos y materiales. Para realizar una Planeación Estratégica que ayude a estar preparados para enfrentar estos desafíos, se pueden implementar los siguientes pasos.

Pregúntate: ¿Qué quiero?, ¿A dónde quiero llegar? ¿Cuál es mi situación actual? Y ¿cómo cierro la distancia entre lo que quiero y lo que tengo? Realiza un análisis situacional (Rodríguez, 2016).

Todo cambio en una organización genera nuevas oportunidades que le permitan lograr una estabilidad y reconocimiento en su mercado, de forma que se pueda alcanzar la competitividad y satisfacción de los clientes a través de la oferta de servicios y productos de excelencia.

\subsection{Etapas}

Para gestionar los recursos disponibles de la organización es necesario establecer una planificación estratégica, tal como lo 
menciona Capriotti (2013). Las etapas radican en los siguientes puntos:

- El análisis del perfil corporativo, por medio del cual se estudia y define la personalidad de la organización (análisis interno), y también se analiza a la competencia y a los públicos de la organización, así como la imagen que tienen cada uno de ellos respecto a la compañía (análisis externo).

- La definición del perfil corporativo, etapa en la que se deberán tomar las decisiones estratégicas dirigidas a definir cuáles son los atributos básicos de identificación de la organización, que permiten la identificación, diferenciación, la referencia y la preferencia.

- La comunicación del perfil Corporativo, por medio de la cual se determinan las posibilidades comunicativas de la organización y se comunica a los diferentes públicos de la compañía el perfil de identificación.

\section{Conclusiones}

Las empresas deben seguir estrategias de planificación que permitan optimizar el uso de recursos tanto humanos $y$ económicos. Por lo tanto, al no aplicar herramientas de gestión estratégica, la empresa tiende a no mantener objetivos acordes a su necesidad actual, por ello, es necesario que se aplique una planificación estratégica corporativa, planteando metas y objetivos que aseguren su éxito empresarial.

\section{Bibliografía}

Álvarez-Hernández de Alba, N., Chongo-Espinosa, D., \& Pérez-Salazar, Y. (2014). La teoría de la gestión financiera operativa desde la perspectiva marxista. Economía y DesarrolloScielo, 161-173. Obtenido de http://scielo.sld.cu/scielo.php? pid=S0252$85842014000100013 \&$ script $=$ sci_arttext\&tlng=pt\#1

Capriotti, P. (2013). Planificación estratégica de la imagen corporativa (Cuarta ed.). Málaga, España: Instituto de Investigación en Relaciones Públicas. Obtenido de http://www.bidireccional.net

Carrasco-Zújar, C., \& PallerolaComamala, J. (2014). Gestión Financiera. Madrid, España: RA-MA S.A.

Castaño-Ramírez, A. (2011). Planeación estratégica en la Pyme de Bogotá. Orientación 
al futuro y evasión de la incertidumbre. Contaduría y administración, (235), 147173.

Flores, B. (2013). Plan Estratégico para el fortalecimiento de la relación Escuela-Comunidad en Educación Básica. Maracaibo, Venezuela.

Gutiérrez-Zarabanda, A. Y. (2013). El contador público dentro de la Gestión Financiera. Bogotá, Colombia. Obtenido de https://repository.unimilitar.ed u.co/.../1/GutiérrezZarabanda AlecyYovana2013.pd

Haro de Rosario, A., \& Rosario-Díaz, J. F. (2017). Gestión Financiera. Almería: edualeditorial Universidad de Almería.

Luna-Carreño, H. (2016). Diseño de un plan estratégico para mejorar la gestión administrativa, financiera, y operativa de la empresa de muebles Cllassic, ubicada en la ciudad Cuenca provincia del Azuay. Tesis de pregrado. Universidad Laica Vicente Rocafuerte de Guayaquil, Ecuador. Obtenido de http://repositorio.ulvr.edu.ec/h andle/44000/722
Morales-Castro, A., \& MoralesCastro, J. (2014). Planeación Financiera. México: Grupo Editorial Patria.

Nava-Rosillón, M. (2014). Análisis financiero: una herramienta clave para una gestión financiera eficiente. Scielo, 56.

Rodríguez, M. (2016). Planificación Esratégica: Fundamentos $y$ herramientas de actuación. Córdoba: Brujas. 